\title{
Synthesis of Polymer Materials by Low Energy Electron Beam II. Effects of Irradiation Dose on EB-Cured Polyurethane-Acrylate Gel Films
}

\author{
Masayuki ANDO and Toshiyuki URYU* \\ Central Research Institute, Dai Nippon Printing Co., Ltd., \\ 1-1-1, Ichigayakaga-cho, Shinjuku-ku, Tokyo 162, Japan \\ *Institute of Industrial Science, University of Tokyo, \\ 7-22-1, Roppongi, Minato-ku, Tokyo 106, Japan
}

(Received September 30, 1986)

\begin{abstract}
The structure and properties of the gel films obtained from the electron beam (EB) solid-state polymerization of urethane-acrylate prepolymer were investigated as functions of EB irradiation dose. The film of a prepolymer which was synthesized by the reaction of poly(butylene adipate)diol, 4,4'-diphenylmethane diisocyanate, and 2-hydroxyethyl acrylate, contained the crystalline phase arising from poly(butylene adipate). Spherulitic texture was observed on the gel film surfaces by scanning electron microscopy. Spherulitic size decreased drastically from 2.5 to $5 \mathrm{Mrad}$ and diminished gradually from 5 to $20 \mathrm{Mrad}$. The crystallinity was reduced with increasing dose, while crystallite size decreased remarkably in the ranges of $0-1$ and 5-15 Mrad. Since the crosslinking density of this EB-cured gel film rose steeply up to $5 \mathrm{Mrad}$ but tended to increase gradually after $5 \mathrm{Mrad}$, it was assumed that these alternations at lower dose (below $5 \mathrm{Mrad}$ ) were due mainly to crosslinking by the terminal acryloyl double bonds, but at higher dose (above $5 \mathrm{Mrad}$ ), was due to the crosslinking relating to polymer backbones under EB heat damage. Such features of the gel films as functions of EB dose resulted in maximum values at $2.5 \mathrm{Mrad}$ on stress at yield.

KEY WORDS Urethane-Acrylate / Electron Beam Solid-State Polymerization / Morphology / Crystallinity / Crystallite Size / Crosslinking Density / Mechanical Strength /
\end{abstract}

High-intensity radiation processing has been developed as one of the alternatives to heat processing from energy-saving, ecological, and economical viewpoints. ${ }^{1}$ Particularly, low energy electron beam (EB) processing is expected to find the industrial application. ${ }^{2}$

EB-curable materials consist mainly of monomer and/or prepolymer with acryloyl or methacryloyl groups. ${ }^{3,4}$ Since the EB polymerization of such materials causes crosslinking and grafting reactions, ${ }^{5}$ the resulting polymer materials are expected to show properties different from heat-cured polymer materials.
In the previous study, we demonstrated that EB-polymerization proceeded with remainment of the morphology of a prepolymer in the solid state. ${ }^{6}$ For that reason, the crystallinity and mechanical strength of EB-cured polyurethane-acrylate film were higher than those of UV-cured film. In this case, the effects of EB dose on the structure and properties of EB-cured polyurethane-acrylate film are of great interest since damage to the crystalline phase and crosslinking by EB are known to occur simultaneously. ${ }^{7}$

In this study, to examine the complicated

\footnotetext{
* To whom all correspondence should be addressed.
} 
behavior of EB solid-state polymerization and obtain structure-regulated materials by EB solid-state polymerization, the effects of EB irradiation dose on the structure and mechanical properties of EB-cured polyurethaneacrylate films were investigated. For this purpose, we prepared a prepolymer by the reaction of poly(butylene adipate)diol with $4,4^{\prime}$ diphenylmethane diisocyanate and 2-hydroxyethyl acrylate. The characterization of EBcured gel films was carried out by scanning electron microscopy (SEM), X-ray diffraction, and tensile testing.

\section{EXPERIMENTAL}

\section{Material}

Urethane-acrylate prepolymer (UA-251M) was synthesized through the two-step reaction according to Scheme $1^{6}$

In the first step, $100 \mathrm{~g}$ of poly(butylene adipate)diol (1, PBAD, Nippon Polyurethane Industry Co., Ltd., the number average molecular weight $\left(\bar{M}_{n}\right)$ of about 2500$)$ were reacted with $20 \mathrm{~g}$ of $4,4^{\prime}$-diphenylmethane diisocyanate (2, MDI) in methyl ethyl ketone (MEK) under a nitrogen atmosphere with di$n$-butyltin-dilaulate as a catalyst to give an isocyanate-capped intermediate (3). In the second step, 3 was reacted with $9.8 \mathrm{~g}$ of 2 hydroxyethyl acrylate (4, HEA) under an oxygen atmosphere in the same flask without recovery. The completion of the reaction was confirmed by the disappearance of the peak due to $\mathrm{N}=\mathrm{C}=\mathrm{O}$ stretching absorption near $2270 \mathrm{~cm}^{-1}$ by IR spectroscopy. The final product (5) was purified by reprecipitation and dried under vacuum.

\section{Irradiation}

UA-251 M films, which were cast from MEK solution and dried under vacuum, were melted in the oven at $80^{\circ} \mathrm{C}$ for $0.5 \mathrm{~h}$ and cooled to room temperature. The thickness was about $40 \mu \mathrm{m}$.

An electro-curtain type accelerator (Energy Science Inc.) equipped with a linear filament was used as a electron beam source. EB irradiation was performed under a nitrogen atmosphere and the irradiation dose ranged from 0.5 to $20 \mathrm{Mrad}$ using a beam current of 1 to $20 \mathrm{~mA}$ at an acceleration voltage of $175 \mathrm{kV}$. The dose rate changed from 1.5 to 30.3 $\mathrm{Mrad} \mathrm{s}^{-1}$ during the change of the dose.

\section{Characterization Methods}

The gel fraction of the polymerized UA$251 \mathrm{M}$ was evaluated by extracting a soluble fraction with tetrahydrofuran (THF) at $25^{\circ} \mathrm{C}$ for $72 \mathrm{~h}$. The insoluble or soluble part was dried under vacuum and weighed to give the gel fraction.

The following measurements were performed using the gel film after extraction with THF to avoid effects of unreacted components.

The percent transmittance at the wavelength of $700 \mathrm{~nm}$ was measured at $25^{\circ} \mathrm{C}$ using a UV/VIS recording digital spectrophotometer

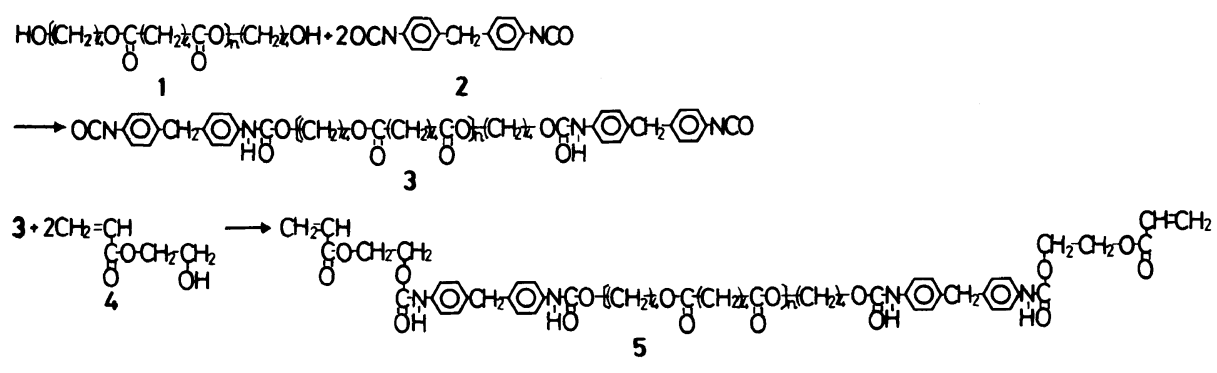

Scheme 1. Synthesis of urethane-acrylate (UA-251M). 1, poly(butylene adipate)diol; 2, 4,4'-diphenylmethane diisocyanate; 3 , isocyanate-capped intermediate; 4 , 2-hydroxyethyl acrylate; 5 , urethane-acrylate prepolymer (UA-251M). 
(Japan Spectroscopic Co., Ltd.).

An Akashi scanning electron microscope (SEM, model ALPHA-30W) was used to observe the morphology of the EB-cured gel film surfaces. Specimens for SEM were prepared by evaporating gold onto the surfaces under vacuum.

X-Ray diffraction patterns were measured using a Geiger Flex (Rigaku Denki Co., Ltd.). The X-ray was nickel-filtered $\mathrm{Cu}-K_{\alpha} \quad(\lambda=$ $0.1542 \mathrm{~nm}$ ) radiation from a sealed tube operated at $35 \mathrm{kV}$ and $16 \mathrm{~mA}$. The degree of crystallinity was calculated using the true diffraction intensity which was defined as the intensity over the line drawn along the base of the diffraction peaks. ${ }^{8}$ The crystallite size $(D)$ was calculated by the Scherrer's equation, ${ }^{8}$

$$
D=0.94 \beta / \lambda \cdot \cos \theta
$$

where $\beta$ indicates the half-height width of the peak of $2 \theta=$ about $21^{\circ}, \lambda$ the wavelength of $X$ ray, and $\theta$ the Bragg's angle.

The crosslinking density was defined as the molecular weight $(M c)$ between crosslinking junctions. $M c$ was calculated by the equation derived from the theoretical state equation for an ideal rubber, ${ }^{9}$

$$
a=\rho \cdot R \cdot T / M c
$$

where a denotes the slope of the low elongation range in the plot of stress against $\left(\alpha-1 / \alpha^{2}\right)$ ( $\alpha$ : the extension ratio) obtained from the stress-strain measurements at $60^{\circ} \mathrm{C}, \rho$ denotes the density, $R$ is the gas constant, and $T$ is the absolute temperature. The stress-strain measurements were carried out using a Tensilon (type UTM-III-100) (Toyo Baldwin Co., Ltd.) with a crosshead speed of $50 \mathrm{~mm} /$ min and a gauge length of $40 \mathrm{~mm}$. Testing sample films were cut $80 \mathrm{~mm}$ long and $10 \mathrm{~mm}$ wide. The engineering stress was calculated as the strength divided by the initial crosssectional area. Mechanical properties were similarly measured at $25^{\circ} \mathrm{C}$.

\section{RESULTS AND DISCUSSION}

\section{Structure of Polyurethane-Acrylate Gel Films}

The urethane-acrylate prepolymer (UA$251 \mathrm{M})$ was readily polymerized by EB irradiation to give both higher molecular weight polymers and densely crosslinked gel, as can be seen from Figure 1. The gel fraction which increased steeply with an increase in dose reached saturation at $5 \mathrm{Mrad}$ irradiation. This indicates that the polymerization by acryloyl double bonds became complete at an irradiation up to $5 \mathrm{Mrad}$. However, even after the $5 \mathrm{Mrad}$ irradiation, the opacity of EB-cured film was enhanced with increasing dose, which was observed from the percent transmittance at $700 \mathrm{~nm}$ of gel films (Figure 1). Although the transmittance of the gel film was less than $5 \%$ in the range of 1 to $5 \mathrm{Mrad}$, it steeply rose in the range of 5 to $15 \mathrm{Mrad}$. The transmittance of the gel film irradiated at $20 \mathrm{Mrad}$ reached more than $20 \%$. It is assumed that this change of the opacity is attributed to the change in the morphology of gel film in dimension of the visible light wavelength since the transmittance decreased with shortening wavelength.

To elucidate the change in the transmittance, the surfaces of gel films were observed by a scanning electron microscope (SEM) (Figure 2).

Four gel films were obtained by the EBirradiation to UA-251M films at 2.5, 5, 10, and

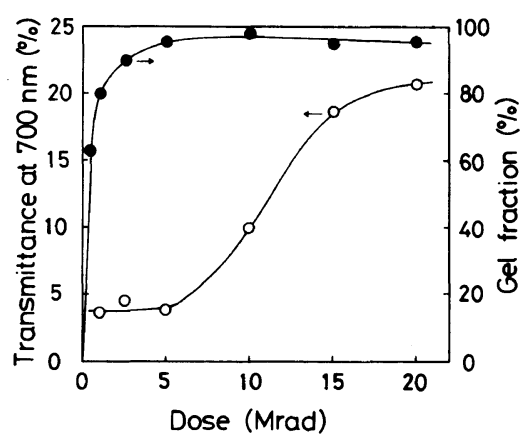

Figure 1. Percent transmittance of gel films obtained from EB-cured UA-251M and gel fraction as functions of dose. 


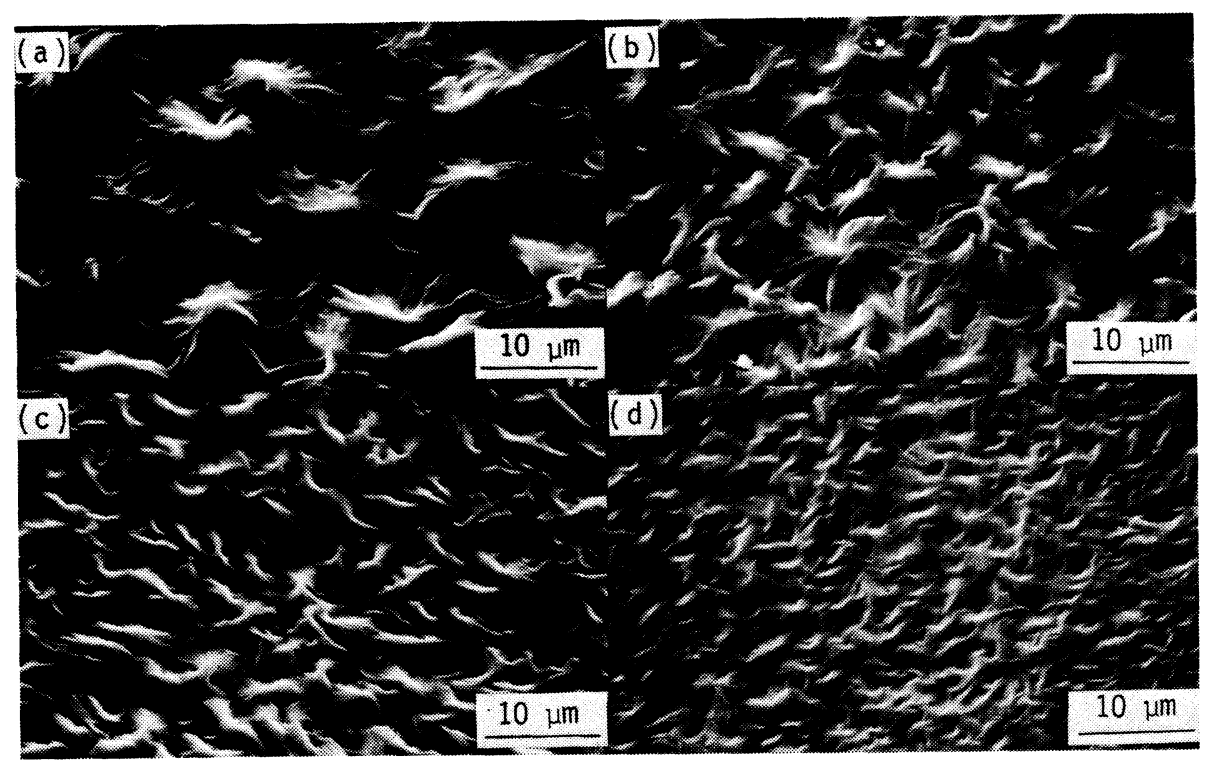

Figure 2. Scanning electron micrographs of gel film surfaces obtained from EB-cured UA-251M. (a), $2.5 \mathrm{Mrad}$; (b), $5 \mathrm{Mrad}$ (c), $10 \mathrm{Mrad}$; (d), 20 Mrad.

$20 \mathrm{Mrad}$. Each gel film proved to have an uneven surface, where the convex part indicates a spherulitic texture originating from a poly(butylene adipate) moiety ${ }^{10}$ and the concave part appears to be due to an amorphous phase. The spherulitic size on the gel film surface decreased drastically in the range from 2.5 to $5 \mathrm{Mrad}$, followed by gradual diminishing in the range from 5 to $20 \mathrm{Mrad}$. In addition, it was observed that the surface of gel film became smooth. Therefore, the increase in transparency proved to be caused by both the decrease in spherulitic size and the increase in smoothness. But, for the film irradiated at less than $5 \mathrm{Mrad}$, the spherulitic size was too large in comparison with the wavelength to change the transparency of the gel film.

Such morphology change was closely related to the crystallinity and crystallite size. Figure 3 shows the effects of dose on the degree of crystallinity and crystallite size. The degree of crystallinity decreased gradually with dose, while crystallite size showed a remarkable decrease at $0-1 \mathrm{Mrad}$ and a gradual decrease at 5-15 Mrad.

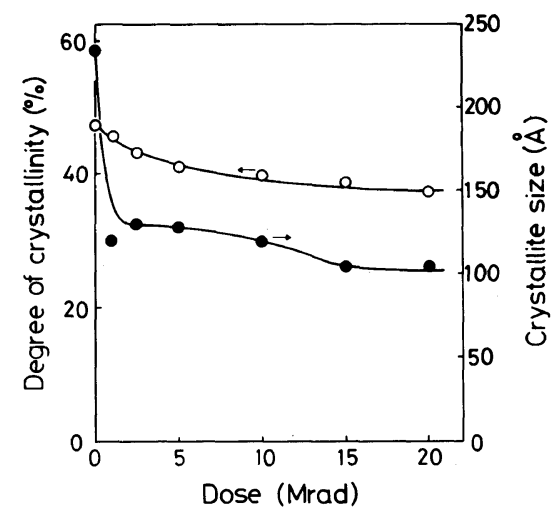

Figure 3. Degree of crystallinity and crystallite size of gel films obtained from EB-cured UA-251A as functions of dose.

Next, the cause for these changes induced by EB irradiation is discussed. When the prepolymer film was made by applying the polymer solution on the base film, evaporation of solvent may have resulted in the crystallization of polymer chains. It is considered that the EB irradiation caused the crosslinking of polymer chains, preventing any change in the morphology. It has been found that the crosslink- 
Table I. Effects of dose rate on structure and properties of EB-cured UA-251M gel films

\begin{tabular}{llcccccc}
\hline Dose & $\left(\mathrm{Mrad}^{2}\right.$ & 5 & 5 & 5 & 10 & 15 & 20 \\
\multicolumn{1}{c}{ Dose rate } & $\left(\mathrm{Mrad} \mathrm{s}^{-1}\right)$ & 7.6 & 15.2 & 30.3 & 15.2 & 22.8 & 30.3 \\
\hline Degree of crystallinity & $(\%)$ & 41.6 & 41.0 & 40.2 & 39.8 & 38.7 & 37.3 \\
Crystallite size & $(\AA)$ & 128 & 120 & 136 & 120 & 105 & 105 \\
Stress at yield & $\left(\mathrm{kg} \mathrm{cm}^{-2}\right)$ & 80.7 & 80.0 & 78.5 & 72.8 & 67.5 & 67.4 \\
Young's modulus $\times 10^{-3}$ & $\left(\mathrm{~kg} \mathrm{~cm}^{-2}\right)$ & 1.48 & 1.46 & 1.44 & 1.43 & 1.42 & 1.32 \\
\hline
\end{tabular}

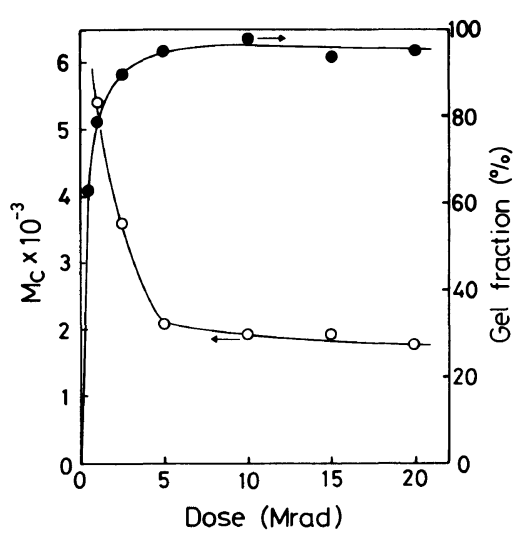

Figure 4. Molecular weight $(M c)$ between crosslinking junctions of gel films obtained from EB-cured UA-251M and gel fraction as functions of dose.

ing junctions formed by $\gamma$-rays prevent the crystalline phase from regenerating when the polymer is cooled from the melt state. $^{11}$ Therefore, it is also assumed in this experiment that the change in surface morphology and decrease in both crystallinity and crystallite size observed after the EB irradiation were predominantly due to the crosslinking structure.

To further clarify this, the molecular weight $(M c)$ between crosslinking junctions defined as the crosslinking density was determined. As shown in Figure 4, $M c$ decreased steeply up to $5 \mathrm{Mrad}$, but tended to decrease gradually after $5 \mathrm{Mrad}$ irradiation. Accordingly, it was found from the $M c$ values that the change in morphology and decrease in crystallite size are closely related to the crosslinking density. Taking into account the steep rise of the crosslinking density at the lower dose, the remarkable decreases in spherulitic and crystallite size are exclusively due to increase in the crosslinking density caused by the reaction of terminal acryloyl double bonds. Particularly, the largest texture at $2.5 \mathrm{Mrad}$ seemed due to the larger $M c$ of the gel film than that of the prepolymer film (Figure 2).

On the other hand, at the higher dose, the gradual decreases in spherulitic and crystallite size may be due to the crosslinking related to polymer backbones. Crosslinking is known to be so difficult in the crystalline region that it occurs mainly in the amorphous region. ${ }^{12}$

Such dose effects are discussed in more detail in the following. Dose increased with dose rate in this experiment and thus the heating and radiation density effects may not be ignored at high dose rates. Consequently, the effects of dose rate on the structure and properties of EB-cured gel films were studied. The results are shown in Table I.

As the dose rate increased from 7.6 to 30.3 $\mathrm{Mrad} \mathrm{s}^{-1}$ with dose from 5 to $20 \mathrm{Mrad}$, the dose rate changed from 7.6 to $30.3 \mathrm{Mrad} \mathrm{s}^{-1}$ at a fixed dose of $5 \mathrm{Mrad}$. Comparing the degree of crystallinity and crystallite size of the resulting gel films with the results from Figure 3, it is evident that the effects of dose rate are much less than those of the dose. But, since a small decrease in the degree of crystallinity is recognized, the effects of dose rate may not be completely neglected. The heat evolution by EB irradiation of more than $15 \mathrm{Mrad}$ or polymerization was significant and should promote the destruction of a crystalline phase into an amorphous phase. ${ }^{12}$ 


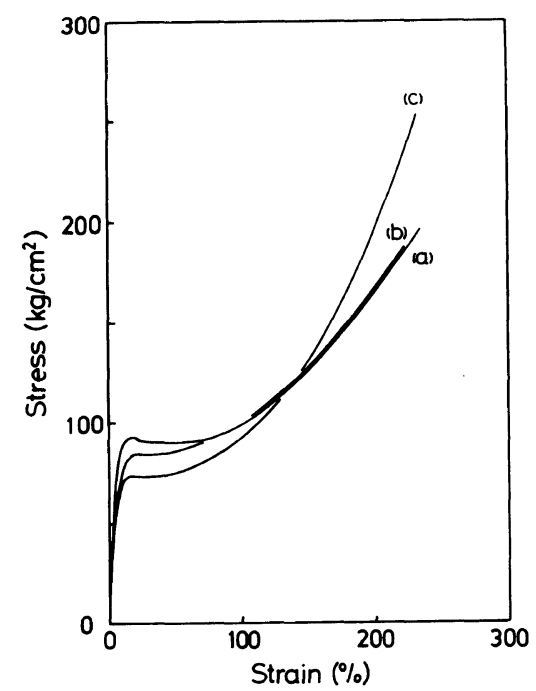

Figure 5. Effects of dose on stress-strain curves at $25^{\circ} \mathrm{C}$ of gel films obtained from EB-cured UA-251M. (a), $2.5 \mathrm{Mrad}$; (b), $5 \mathrm{Mrad}$; (c), $10 \mathrm{Mrad}$.

\section{Mechanical Properties of EB-Cured Poly- urethane-Acrylate Gel Films}

The effects of the structure of EB-cured UA$251 \mathrm{M}$ gel film on its mechanical properties were studied. Representative stress-strain curves of the gel films are shown in Figure 5. It was recognized that the EB irradiation dose affected the stress at yield, Young's modulus, and tensile strength. To elucidate the effects of dose on such mechanical properties, these values and the elongation at break were plotted against dose. The results are shown in Figures 6, 7, and 8.

It is noteworthy that the stress at yield and Young's modulus indicated maximum values at 2.5 Mrad (Figures 6 and 7). These maxima can be explained in terms of the morphology of polymer chains and crosslinking density. In general, an increase in crystallinity enhances the stress at yield and Young's modulus. But, for polypropylene, it has been reported that in a narrow range of crystallinity, the increase in spherulitic size leads to a decrease in stress at yield and Young's modulus. ${ }^{13,14}$ Since in highly crystalline polymers, the stress concentrates on the boundaries between large spher-

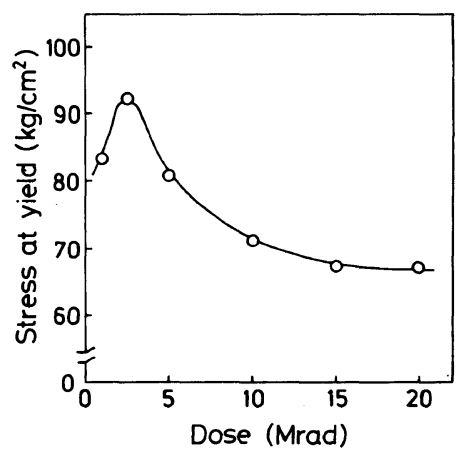

Figure 6. Stress at yield of gel films obtained from EBcured UA-251M as a function of dose.

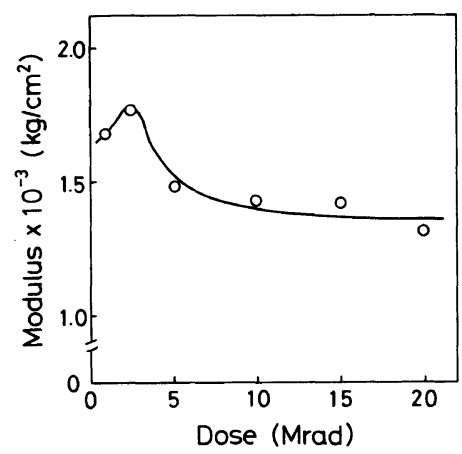

Figure 7. Young's modulus of gel films obtained from EB-cured UA-251M as a function of dose.

ulites where few tie molecules exist, the abovementioned decrease in mechanical strength takes place. ${ }^{15}$ In this system, below $2.5 \mathrm{Mrad}$, the EB-cured gel film showed phenomena analogous to polypropylene.

When EB irradiation is sufficient to cause crosslinking, the spherulites of EB-cured gel film must be connected by tie molecules formed by crosslinking between poly(butylene adipate) moieties. However, the crosslinking between crystalline regions did not take place in the EB irradiation below $2.5 \mathrm{Mrad}$. Thus, the gel film obtained below 2.5 Mard was low in stress at yield and modulus.

In contrast, above $2.5 \mathrm{Mrad}$, in spite of an increase in crosslinking density, crystallinity decreased with increasing dose, which might induce a gradual lowering of stress at yield and modulus. A rapid decrease in spherulite size 


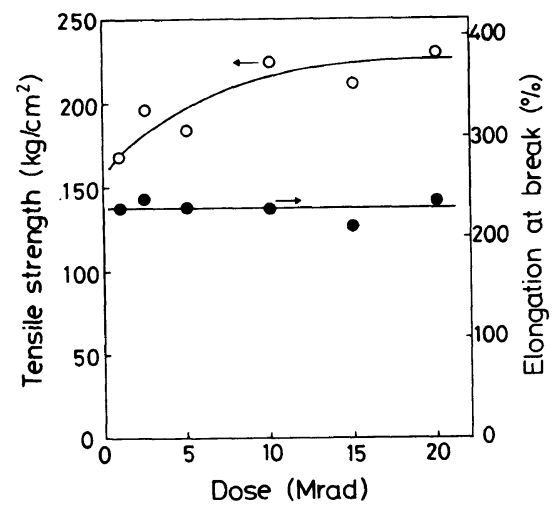

Figure 8. Tensile strength and elongation at break of gel films obtained from EB-cured UA-251M as functions of dose.

was observed in the $2.5-5 \mathrm{Mrad}$ region, as seen in Figure 2(a) and (b).

Moreover, it was confirmed from the stress at yield and modulus that the dose effect is far larger than the dose rate effect, as shown in Table I. This also indicates that the dose predominantly regulates the structure of EBcured gel films.

As shown in Figure 8, the features of tensile strength were different from those of the above mechanical properties. That is to say, tensile strength increased with increasing dose from 1 to $20 \mathrm{Mrad}$. This increase seems to be influenced strongly by crosslinking between poly(butylene adipate) chains.

On the other hand, the elongation at break was constant and independent of dose. Usually, elongation depends mainly on molecular weight between crosslinking junctions. ${ }^{16}$ The elongation in this experiment indicated unexpectedly a constant value. This is explained as follows. In the case of low dose, the break of sample film might occur due to incomplete elongation of folded polymer chains because of an insufficient amount of tie molecules. At high dose, the molecular weight of the polymer chains in crystalline regions related to elongation did not depend on dose, because crosslinking was induced at the ends of poly(butylene adipate) chains existing in amorphous regions not related to elongation. As a result, EB-induced crosslinking with retainment of spherulitic texture produced unique polymer film with high mechanical properties. The appropriate selection of EB irradiation dose lead to higher mechanical properties.

\section{REFERENCES}

1. G. Pasternack, Radiat. Curing, 8(11), 14 (1981).

2. D. W. Mabrey and S. Surber, Radiat. Curing, 11(2), 2 (1984).

3. F. S. Stowe, Mod. Paint Coatings, 72(6), 43 (1982).

4. E. Kraiman, Radiat. Curing, 11(2), 8 (1982).

5. R. Takiguchi and T. Uryu, Radiat. Phys. Chem., 25, 475 (1985).

6. M. Ando and T. Uryu, Polym. Prepr. Jpn., 35, 358 (1986).

7. L. H. Wadhwa and W. K. Walsh, J. Appl. Polym. Sci., 27, 591 (1982).

8. The Society of Polymer Science, Japan, Ed., "Kobunshi Sokuteiho," Vol. 1, Baifukan, Tokyo, 1973.

9. L. E. Nielsen, J. Macromol. Sci., Rev. Macromol. Chem., C3, 69 (1969).

10. E. Joseph, G. Wilkes, and K. Park, J. Appl. Polym. Sci., 26, 3355 (1981).

11. M. Narkis, S. Sibony-Chaouat, A. Siegmann, S. Shkolnik, and J. P. Bell, Polymer, 26, 50 (1985).

12. M. Ando and T. Uryu, J. Appl. Polym. Sci., in press.

13. L. S. Remaly and J. M. Schultz, J. Appl. Polym. Sci., 14, 1871 (1970).

14. L. Barish, J. Appl. Polym. Sci., 6, 617 (1962).

15. C. F. Hammer, T. A. Koch, and J. F. Whitney, J. Appl. Polym. Sci., 1, 169 (1959).

16. W. Oraby and W. K. Walsh, J. Appl. Polym. Sci., 23, 3227 (1979). 